\title{
Int-DWTs: Simplificações Algébricas para Aumento de Exatidão em Transformadas Wavelets
}

\author{
Vinícius R. dos Santos, Renata H. S. Reiser, Maurício L. Pilla \\ Mestrado em Ciência da Computação, PPGC, CDTEC, UFPel, \\ 96010-000, Rua Gomes Carneiro, 1, Campus Anglo, Pelotas, RS \\ E-mail: \{vrdsantos, reiser, pilla\}@inf.ufpel.edu.br
}

\author{
Alice Kozakevicius \\ Departamento de Matemática, PPGI, LANA \& GMICRO, UFSM \\ 97-105-900, Campus Camobi, Santa Maria, RS \\ E-mail: alicek@ufsm.br.
}

\section{RESUMO}

Palavras-chave: Lógica Fuzzy, Matemática Intervalar, Implicações Fuzzy

A matemática intervalar vem sendo empregada no tratamento da incerteza dos resultados aproximados em algoritmos numéricos da computação científica, onde os valores incertos são armazenados através de intervalos, cujos extremos são pontos flutuantes. Pela aplicação de conceitos da teoria intervalar, viabiliza-se a elaboração de algoritmos autovalidáveis, com controle automático para o limite dos erros inerentes aos processos numéricos de TDWs (Transformadas Discretas Wavelet) em sistemas computacionais.

A principal meta deste projeto consiste no desenvolvimento de extensões intervalares de TDWs, considerando a família de funções wavelets ortonormais de Daubechies, funções Spline-Wavelets [Stollnitz et al. 1995a] e Wavelets interpolatórias [J.M. De Villiers 2003]. Os algoritmos originais de cada uma das transformações são o ponto de partida para o desenvolvimento de bibliotecas contendo suas extensões intervalares.

Em uma primeira etapa foi desenvolvida a biblioteca Int-Haar [dos Santos et al. 2013] com a extensão intervalar das transformadas de Haar 1D e 2D [Stollnitz et al. 1995b]. Através deste estudo preliminar, observou-se que uma questão chave para a obtenção de extensões intervalares competivivas é a possibilidade de se introduzir simplicações algébricas a fim de se eliminar o cálculo de valores irracionais. A avaliação da otimização implementada é realizada utilizando Interval, estrutura de dados de alta precisão contida na biblioteca C-XSC para representação de intervalos reais.

De acordo com os algoritmos originais para a transformada de Haar [Stollnitz et al. 1995b] estudados inicialmente, durante a transformação normalizada o erro é gerado e propagado por todos os níveis de decomposição e composição dos dados. A otimização atua neste processo, separando-o em duas etapas, que correspondem a dois procedimentos complementares.

Durante a primeira etapa os dados são decompostos utilizando a abordagem não normalizada, que não implica em erro de cálculo [dos Santos et al. 2013]. A segunda etapa transforma os coeficientes resultantes utilizando os fatores de normalização, gerados pela regra $R_{N}^{1}$.

Os procedimentos para a execução da TWH bidimensional utilizam do mesmo princípio apresentado no parágrafo anterior. Porém, nesta extensão fora desenvolvido uma segunda regra, denominada neste artigo como $R_{N}^{2}$, para os procedimentos bidimensionais.

As regras para cálculo dos fatores de normalização são descritas como:

$$
R_{N}^{1}=2^{\frac{-j}{2}} \quad ; \quad R_{N}^{2}=2^{\frac{-\left(j^{\prime}+j^{\prime \prime}\right)}{2}}
$$


onde que $0 \leq j, j^{\prime}, j^{\prime \prime} \leq\left(\log _{2} n\right)-1, n$ indica a ordem da matriz e $j, j^{\prime}$ e $j^{\prime \prime}$ são os níveis de aplicação da TWH.

\section{TESTES E RESULTADOS}

De acordo com os resultados apresentados na Tabela 1 para a TWH-intervalar, os algoritmos desenvolvidos apresentaram resultados mais exatos do que os da literatura, motivando o desenvolvimento de otimizações e extensões intervalares para as demais transformadas. É importante verificar que os cálculos de $\sqrt{2}$ para o novo método não-padrão para a Int-Haar não precisaram ser executados, devido à simplificação algébrica implementada.

Tabela 1: Erros de cálculo da Int-Haar gerados pelos algoritmos da literatura e os desenvolvidos no trabalho, utilizando a abordagem normalizada.

\begin{tabular}{|l|l|l|}
\hline Metodologias & Decomposição & Composição \\
\hline Método Padrão Novo & $3.492460 \mathrm{E}-010$ & $6.705523 \mathrm{E}-008$ \\
Método Padrão Original & $7.916242 \mathrm{E}-008$ & $4.671514 \mathrm{E}-006$ \\
Método Não-Padrão Novo & 0.000000 & 0.000000 \\
Método Não-Padrão Original & $7.171184 \mathrm{E}-008$ & $1.819804 \mathrm{E}-006$ \\
\hline
\end{tabular}

\section{CONCLUSÃO}

Este trabalho apresentou a implementação da versão intervalar do algoritmo da TWH obtendo bons resultados quanto à análise do erro, ou seja, as otimizações viabilizaram o aumento na exatidão dos cálculos.

Como as transformadas wavelets são apropriadas para a análise de dados em contextos nos quais as escalas de representação são relevantes para o problema, o ganho de exatidão obtido com as simplificações propostas neste trabalho representam uma contribuição significativa para esta área de pesquisa.

Na continuidade, busca-se a extensão da biblioteca para suporte a programação paralela, utilizando OpenMP ou o uso de paralelização massiva em GPGPUs (General-Processing Graphics Processing Units).

\section{Referências}

[dos Santos et al. 2013] dos Santos, V., Pilla, M., Reiser, R., and Kozakevicius, A. (2013). Inthaar: Extensão intervalar da transformada de haar. In Escola regional de Alto Desempenho, ERAD 2013, pages 167-170, POA. SBC.

[J.M. De Villiers 2003] J.M. De Villiers, K.M. Goosen, B. H. (2003). Dubuc-deslauriers subdivision for finite sequences and interpolation wavelets on an interval. SIAM J Applied Maths, 35:423-452.

[Stollnitz et al. 1995a] Stollnitz, E. J., DeRose, T. D., and Salesin, D. H. (1995a). Wavelets for computer graphics: A primer - part 2. IEEE Computer Graphics and Applications, 15:76-84.

[Stollnitz et al. 1995b] Stollnitz, E. J., DeRose, T. D., and Salesin, D. H. (1995b). Wavelets for computer graphics: A primer, part 1. IEEE Computer Graphics and Applications, 15(3):7684. 